\title{
Die Nullzinsdebatte - wenn Tore nicht mehr zählen
}

Nach der EZB-Entscheidung, den Refinanzierungszins auf Null zu senken und das Anleihekaufprogramm auszuweiten, gab es wieder die bekannten Bilder von der Geldflut, die Warnungen vor Risiken und Experimenten sowie die Charakterisierung der Geldpolitik als Irrfahrt sowie Mandatsverletzung. Das Votum fiel - von wenigen Ausnahmen abgesehen - so negativ aus, dass man sich fragt, wie sich die EZBPolitik noch ernsthaft für sachgerecht erklären lässt. Vielleicht gelingt es mit einem Vergleich, auch wenn bekanntlich jeder Vergleich hinkt. Man stelle sich vor, es ist Samstagabend, Sportschauzeit. Ausführlich werden die Zuschauer darüber informiert, welche Trikots die Mannschaften trugen, wie viele gelbe Karten es gab, und welche Mannschaft wie oft in Ballbesitz war. Nur das, worauf es im Fußball ankommt, Tore und Ergebnisse, bleibt ausgespart.

Als die EZB am 10. März 2016 ihre Entscheidung bekannt gab, fiel die Kommentierung überwiegend genauso aus. Alles wurde erwähnt: dass die Geldpolitik Überhitzungen am Immobilien- und Aktienmarkt fördert, den Sparer enteignet, eine fiskalische Umverteilungspolitik betreibt, überschuldete Staaten und Zombiebanken subventioniert und Wirtschaftsreformen verschleppt. Nur worauf es der Geldpolitik ankommt, Preisstabilität, wurde gar nicht oder nur im Nebensatz erwähnt. Preisstabilität ist aber das primäre Ziel der Geldpolitik. Ist also eine Zinssetzung notwendig, um Preisstabilität zu sichern, muss die EZB diese durchführen, selbst wenn der Sparer darunter leidet, die Zombiebank davon profitiert, und ein Land deshalb Wirtschaftsreformen verschiebt. Diese Eindeutigkeit in der Zielfestlegung, verankert im Maastrichter Vertrag, ist der herrschenden Geldtheorie und dem geldpolitischen Konsens geschuldet. Danach kann die Geldpolitik mittel- und langfristig nur Preisstabilität und keine anderen Ziele erreichen. Von ihr zu verlangen, andere Ziele zu verfolgen, etwa einen positiven Realzins oder Anreize für Reformen, widerspricht allem, wofür deutsche Ökonomen und Politiker über Jahrzehnte gekämpft haben.

Bisher bestand Konsens, dass Preisstabilität als eine Inflationsrate knapp unter 2\% in der mittleren Frist definiert ist. Es gab auch keine Zweifel darüber, dass die Geldpolitik bei entschlossenem Handeln Preisstabilität gewährleisten kann, indem sie bei Inflation den Zins erhöht, sowie den Zins senkt, im Notfall auch Anleihen kauft, wenn das Ziel von unten verfehlt wird. Auf dieser Grundlage haben wir eine unabhängige Notenbank geschaffen: damit sie jenseits politischer Erwägungen ihre Instrumente dafür einsetzt, Preisstabilität zu erreichen. Entsprechend wurde früher nach geldpolitischen Entscheidungen darüber diskutiert, ob die Notenbank alles getan hat, um Preisstabilität zu sichern. Nebenwirkungen der Geldpolitik blieben dagegen außen vor bzw. wurden vor allem von Beobachtern thematisiert, die der EZB und vor 1999 der Bundesbank (woran sie sich anscheinend nicht mehr erinnern mag) nahelegten, es mit dem Ziel Preisstabilität nicht so genau zu nehmen. Mit anderen Worten: Die Nebenwirkungen spielten für die Beurteilung der Geldpolitik ungefähr die gleiche Rolle wie die Zahl der gelben Karten im Fußball: relevant, aber nicht entscheidend.

Die Inflationsrate im Euroraum ist praktisch Null. Zum letzten Mal lag sie um die Jahreswende 2012/2013 bei 2\%. Das ist eine Zielverfehlung in der "mittleren Frist“. EZB-Kritiker sagen, dass das nicht stimmt, und verweisen auf den Ölpreis, der die Inflationsrate nach unten treibt. Allerdings: In 78 der letzten 87 Monate bewegte sich auch die Kerninflationsrate, bei der der Ölpreis herausgerechnet wird, unter 1,9\%; im

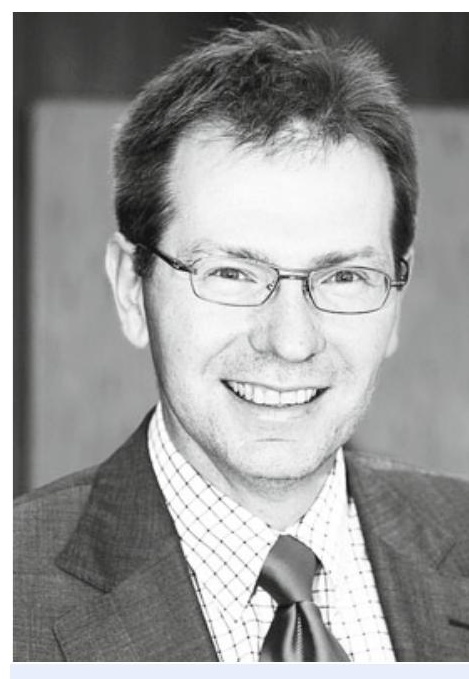

Adalbert Winkler ist

Professor für International and Development Finance an der Frankfurt School of Finance \& Management in Frankfurt am Main. 
Durchschnitt der letzten drei Jahren betrug sie 1\%. Somit ist klar: Eine kritische geldpolitische Diskussion müsste sich vor allem mit der Frage beschäftigen, wann und wie die EZB wieder ihr Ziel erreichen kann.

Das ist aber nicht die Diskussion, die die EZB-Kritiker führen. Denn dann müssten sie ausführen, dass die Geldpolitik nicht locker genug ist, oder andere Politikbereiche, z.B. die Fiskalpolitik, auffordern, die Geldpolitik zu unterstützen. Sie würden sich also selbst widersprechen. Deshalb wählen sie den Weg, das Ziel der Geldpolitik wahlweise auszublenden, zu relativieren, neu zu definieren oder als nicht mehr erreichbar anzusehen. Damit wird zweierlei erreicht: Erstens gerät ihre falsche Analyse der Jahre 2012/2013, als sie vor den inflationären Gefahren einer lockeren Geldpolitik warnten, in Vergessenheit. Zweitens können sie die negativen Nebenwirkungen der Geldpolitik ins Zentrum der Debatte rücken, ohne Auskunft darüber geben zu müssen, wie die EZB Preisstabilität unter den derzeit herrschenden Bedingungen erreichen soll. Es ist eine bequeme Position, weil diese Nebenwirkungen zurzeit besonders den deutschen Sparer betreffen. Dass der deutsche Staat und Steuerzahler von den niedrigen Zinsen profitieren, bleibt dagegen unerwähnt.

Damit keine Missverständnisse entstehen: Es mag gute Gründe geben, über Ziele der Geldpolitik zu diskutieren. Die Geldpolitik hat derzeit große Schwierigkeiten, Preisstabilität zu sichern, und es werden ungewöhnliche Maßnahmen getroffen, um das Ziel zu erreichen. Allerdings sollte man diese Diskussion nicht klammheimlich, sondern offen führen. Dann würde sich schnell zeigen, warum wir der Geldpolitik das Ziel Preisstabilität übertragen haben. Und gleichzeitig müssten die Kritiker die Konsequenzen benennen, die entstehen, wenn man die Geldpolitik von diesem Ziel entbinden würde. Genau deshalb sind sie nicht bereit, diese Diskussion ernsthaft anzustoßen. Die Diskussion wäre nämlich ungefähr so revolutionär, wie wenn man bei der Reform der FIFA auch darüber sprechen würde, dass in Zukunft nicht mehr die Mannschaft gewinnen soll, die die meisten Tore schießt.

Beim Fußball herrscht Konsens über das Ziel: Das Runde muss ins Eckige. In der geldpolitischen Debatte kündigen die EZB-Kritiker diesen Konsens über das Ziel auf. Sie werfen nämlich der Mannschaft nicht vor, dass sie Spiele verliert, sondern dass sie sich darum bemüht, Spiele zu gewinnen, indem sie hingebungsvoll kämpft und aggressiv in die Zweikämpfe geht. Denn damit riskieren die Spieler, nach dem Spiel total erschöpft zu sein bzw. sich zu verletzen. Und früher genügte es doch auch, 60 Minuten konzentriert zu spielen, um zu gewinnen. Bei den Fußballfans kommen solche Überlegungen, die eine Niederlage mit der Vermeidung negativer Nebenwirkungen rechtfertigen, nicht gut an - selbst in Testspielen nicht. Dies durfte man jüngst nach der 2:3-Niederlage gegen England und den „Larifari“-Äußerungen von Thomas Müller erleben. Im Fußball zählen Tore. Und deshalb wird einer Mannschaft, die „über den Kampf zum Spiel" findet, auch nicht vorgeworfen, dass sie hohe Risiken eingeht, ein gefährliches Experiment wagt, sich auf eine Irrfahrt begibt und ihr Mandat verletzt.

In der Debatte der letzten Jahre ist es den EZB-Kritikern gelungen, den Bürgern zu suggerieren, dass es bei Geldpolitik nicht um Preisstabilität geht, sondern um Sparer und Zombiebanken, die Vermeidung von Staatsanleihekäufen und Anreize für Reformen. Und indem sie der EZB vorwerfen, bei diesen neu formulierten Zielen zu versagen, erzeugen sie den „Wutbürger“, der den Eindruck bekommen muss, dass „die da oben“ mal wieder „machen, was sie wollen“. Dabei sind es die Kritiker, die sich so

Adalbert Winkler

Frankfurt School of Finance \&

Management

a.winkler@fs.de verhalten, indem sie beliebig und ohne Begründung das Ziel Preisstabilität ignorieren oder neu definieren. Sobald man wieder Preisstabilität in den Mittelpunkt der Analyse stellt, muss man aber feststellen: Selbst wenn man über Einzelheiten streiten kann, die EZB macht nichts anderes, als das Ziel anzustreben, das ihr vorgegeben wurde. 